\title{
Theoretical Modelling of Stock Price Dynamics in Stock Markets Using A Phynance Approach
}

\author{
Leonard Mushunje \\ Midlands State University, Gweru, Zimbabwe
}

\begin{abstract}
In this paper we present time to time price dynamics associated with stock assets within stock markets. Our conjecture was that, stock prices are stochastic and time variant as such they do attain and possess different values from time to time. We then centrally aimed to model this old way phenomenon of stock price dynamics using a distinct model from the physics field so as to substantially expose the core idea of phynance on an open academic space. We used the two-form of Schrödinger wave Equation (SWE) to fully model our core study. We derived the time part and space (market) value functions for stock assets from the SWE. Meaning that, we managed to derive the time function measuring the time intervals taken by stock assets in the market space and the market value function which gives out the value of stocks without any time factor. Our results suggested that, stock price dynamics can well be modelled and presented using both time independent Schrödinger equation (TISE) and time dependent Schrödinger equation (TDSE) with traceable stock price and time changes. This supported our conjecture and our model proposition as stock prices are traditionally known to be stochastic in nature and normally they are non-stationary. As such we safely concluded that, physics indeed play important roles when modelling finance problems, hence phynance should be well credited in finance as it presents fruitful and powerful abstractions of the real time happenings in the financial markets.
\end{abstract}

Keywords: stock price dynamics, phynance, Schrodinger wave Equaqtion, stochastic

\section{Introduction}

Over the years, stock price behaviour investigations and analysis were carried out on a more traditional way. The ideological approaches used were mostly rich in mathematical expressions. Application of econophysics and phynance in the field of financial economics appears to be a less concentrated and researched area despite all its great value. On the other hand, stochastic volatility, subordination, and fluctuations in stock trades are an important area in particular that most investors and market players do worry about. The stock price $S_{t}$ is a stochastic time series variable meaning that it depends highly on time and it is usually non-stationary. The non-stationary of these prices forms the basis of this paper and we are going to present and to model it in an unique way. This phenomenon is commonly characterized by the probability distribution $\mathrm{P} \Delta \mathrm{t}(\mathrm{x})$ of detrended log-returns $\mathrm{S}_{\mathrm{r}}=\ln \left(\frac{\mathrm{S}_{\mathrm{t}_{2}}}{\mathrm{~S}_{\mathrm{t}_{1}}}\right)-\mu \Delta \mathrm{t}$ where the time inter-value $\Delta \mathrm{t}=\mathrm{t}_{2}-\mathrm{t}_{1}$ is called the

Leonard Mushunje, B.Sc. in Mathematics, Department of Applied Mathematics and Statistics, Midlands State University, Gweru, Zimbabwe.

Correspondence concerning this article should be addressed to Leonard Mushunje, Department of Applied Mathematics and Statistics, Midlands State University, 9055 Senga Road, Gweru, Zimbabwe. Email address: leonsmushunje@gmail.com 
time lag or time horizon, and $\mu$ is the average growth rate. For a simple multiplicative (geometric) random walk, the probability distribution is Gaussian: $\mathrm{P} \Delta \mathrm{t}(\mathrm{x}) \propto \exp \left(-\frac{\mathrm{x} 2}{2 \mathrm{v} \Delta \mathrm{t}}\right), \mathrm{re} \mathrm{v}=\sigma^{2}$ is the variance, and $\sigma$ is the volatility. Many stochastic models have been widely applied but mainly inclined to statistical probability models. This paper in particular is going to model price dynamics using the Schrödinger equation (SE). We shall analyse our model using techniques from ordinary differential equations and presented our solutions. Contextual interpretations are as well presented with some valuable lessons on board. The notion of stock price dynamics is not new at all. Reviewed literature does speak and present a number of related works. Some of them include, Kunsch, Theys, Chevalier, and Iacopetta (2013) who studied a classical model for stock price dynamics. They tested that richer patterns can be generated in the continuous case using System Dynamics (SD) using finite difference equations. Other related works on stock price dynamics are found in papers by Creedy and Martin (Eds.,1994) and Heij, Schumacher, Hanzon, and Praagman (Eds., 1997). Additionally, Ventana Systems (1997) has been used in the present case for computing the stock price evolution in different scenarios. Such modelling and applications have proved to stand a strong practical impact in the world of finance. Moreover, Caginalp and Desantis (2009) presented a methodology to study a data set of 119,260 daily closed-end fund prices using mixed-effects regressions with the objective of understanding price dynamics. They found that there is a strong statistical support that relative price change depends significantly on: (i) the recent trend in a nonlinear manner; (ii) recent changes in valuation; (iii) recent changes in money supply (M2); (iv) longer-term trend; (v) recent volume changes; and (vi) proximity to a recent high price. From all the existing literature, we found no related or direct application of the Schrödinger model in analysing these price dynamics phenomena. Rather we instead found our model in literature but in other terms than financial. For example Christianto (2014) reviewed some inadequacies of the Schrödinger equation and then discussed the George Shpenkov's interpretation of classical wave equation. There was no application there. However, we noted that, only the application of phynance using Schrödinger wave equation is new despite other applications within the physics field such as its application to arrive at hydrogen energy levels. David and Fatigun (n.d.), discussed the Schrodinger's model and its applications. They showed that the time-independent operators correspond to the observables of the quantum system. Also, from the Schrodinger model, they proved that the model can be used to represent physical quantities such as quanta energy, quanta momentum, and harmonic oscillator. Interestingly, there was no possible application of the model in finance. This then defines the motivation of our paper. Their results also showed that operators are very useful tools for the representation of the eigen-functions of the harmonic oscillator. We in this short paper are going to empower and signify the idea of phynance through a massive and unique application of the Schrödinger wave model (SWM) to stock price dynamics. As the theoretical and interdisciplinary, interpretation and analysis in financial terms is however going to be done. The rest of the paper proceeds as follows: Section 2 took a review of existing price dynamics models, Section 3 presented the methodology including the model used, Section 4 explained the results and findings, discussions and conclusions were presented in Section 5.

\section{Model}

In this particular study, we conjectured that, stock assets of any volume level can be better modelled using appropriate physics models and in particular we put forward Schrödinger's wave equation as our model. We theoretically used the model to present the time to time behaviour of stock assets. The model goes as: 


\section{Schrodinger Wave Equation}

This model was developed by Erwin Schrödinger (1925). The model was formulated in the context of quantum physics using the basis of de-Broglie wave and plank's quantum theory. As well Maxwell's wave equation and the Hamiltonian wave equation were greatly consulted to fully present the model. The bottom line was on formulating a powerful wave equation to be used in appropriate arenas of physics. In this study, we considered the SE in a three-dimensional space. This transitionally implies an open and complete market space from where stock prices can take any direction and value. There are two forms of Schrödinger equations that are time dependent and independent wave models. We shall consider both of them and make a comparative analysis to determine the best one that fully describes stock price behaviour. Our comparison shall be based on the underlying idea from stochastic nature of stock prices. However, these models are to be derived in this paper as part of our contribution. Now the Maxwell's wave equation is given as:

$$
\psi=\frac{1}{\mathrm{u}^{2}}\left(\frac{\partial^{2} \psi}{\partial \mathrm{t}^{2}}\right)
$$

The above equation shall greatly help us to derive both time independent and dependent Schrödinger equations which are of interest to us. The equation (1) is the building block of the SWE. Combining our idea with that of Faisal (2016), the original equation is given as:

$$
\frac{\mathrm{i} \overline{\mathrm{h}} \psi(\mathrm{x}, \mathrm{y}, \mathrm{z}, \mathrm{t})}{\partial(\mathrm{x}, \mathrm{y}, \mathrm{z}, \mathrm{t})}=-\frac{\overline{\mathrm{h}}^{2}}{2 \mathrm{~m}} \nabla^{2} \psi(\mathrm{x}, \mathrm{y}, \mathrm{z})+\mathrm{U}(\mathrm{x}, \mathrm{y}, \mathrm{z}, \mathrm{t}) \psi(\mathrm{x}, \mathrm{y}, \mathrm{z}, \mathrm{t})
$$

where, $\nabla^{2}=\frac{\partial^{2}}{\partial \mathrm{x}^{2}}+\frac{\partial^{2}}{\partial \mathrm{y}^{2}}+\frac{\partial^{2}}{\partial \mathrm{z}^{2}}$ and it define the Laplacian operator and other variables are defined in Table1 below.

Before deriving anything, we shall start by contextualizing our model from physics to finance. We shall then derive the solutions of the model using ordinary differential equation approaches and physics properties. This is among the key contributions for this paper. Then we shall further interpret the obtained results and contextualize them in financial terms.

\section{Variable Definition and Application to Stock Markets}

We shall define the variables making up the model in physics terms and in financial terms as below.

Table1

Variable Definition and Application to Stock Markets

\begin{tabular}{ll}
\hline Variable meaning in physics & Variable in stock markets. \\
\hline $\mathrm{m}$ (mass of the particle under study) & Stock volume \\
$\mathrm{E}$ (energy of the particle) & Resistance function \\
$\psi(\mathrm{x})$ is the wave function in $\mathrm{x}$ & Trend function (normally non-linear) \\
$\mathrm{U}(\mathrm{x})$ is the particle potential in $\mathrm{x}$ & Stock volatility function \\
$\mathrm{h}$ is the reduced plank constant & Market money supply \\
$\frac{\partial^{2}}{\partial \mathrm{x}^{2}}$ is the second order partial derivative & The second order partial change of prices in the market \\
\hline
\end{tabular}

Note. $\mathrm{E}$ and $\mathrm{m}$ are arbitrary constants.

From the above illustrated table, all the variables of interest are indicated. The interdisciplinary between finance and physics in terms of our study and modelling approach used is clearly explored. Before and without and further calculus, one can safely have an appreciation of phynance. 
Concerning our models, the three-dimensional case is representing the unlimited and unrestricted space for stock price dynamics. This means that, in the market it is possible for stock assets to take any value and to move in any direction provided no other constraining factors. As such we shall stick to that version.

\section{Results and Findings}

We shall derive our solutions using both mathematical and some physical properties. From the Schrodinger Wave Equation (SWE) we shall solve the equation procedurally to derive the time and the market space value functions. This goes as:

From Equation (2), we have,

$$
\frac{i \bar{h} \psi(x, y, z, t)}{\partial(x, y, z, t)}=-\frac{\bar{h}^{2}}{2 m}\left(\frac{\partial^{2} \psi(x, y, z, t)}{\partial\left(x^{2} y^{2} z^{2}\right)}\right)+U(x, y, z, t) \psi(x, y, z, t)
$$

Substituting $\psi(x, y, z, t)$ with XYZT, we get,

$$
\frac{\mathrm{ih} \partial(\mathrm{T})}{\partial \mathrm{t}}=-\frac{\overline{\mathrm{h}}^{2}}{2 \mathrm{~m}}\left(\frac{\mathrm{T} \partial^{2}(\mathrm{XYZ})}{\partial\left(\mathrm{x}^{2} \mathrm{y}^{2} \mathrm{z}^{2}\right)}+\mathrm{UXYZT}\right.
$$

Now, dividing (6) by XYZT, we obtain,

$$
\frac{1}{\mathrm{~T}}\left(\frac{\mathrm{i} \overline{\mathrm{h}} \partial(\mathrm{T})}{\partial \mathrm{t}}\right)=-\frac{\overline{\mathrm{h}}^{2}}{2 \mathrm{~m}}\left(\frac{1}{\mathrm{XYZ}}\left(\frac{\partial^{2}(\mathrm{XYZ})}{\partial\left(\mathrm{x}^{2} \mathrm{y}^{2} \mathrm{z}^{2}\right)}\right)+\mathrm{U}\right.
$$

We then use the splitting rule in order to get the time dependent and independent equations. This follows as:

$$
\frac{1}{\mathrm{~T}}\left(\frac{\mathrm{i} \overline{\mathrm{h}} \partial(\mathrm{T})}{\partial \mathrm{t}}\right)=-\frac{\overline{\mathrm{h}}^{2}}{2 \mathrm{~m}}\left(\frac{1}{\mathrm{XYZ}}\left(\frac{\partial^{2}(\mathrm{XYZ})}{\partial\left(\mathrm{x}^{2} \mathrm{y}^{2} \mathrm{z}^{2}\right)}\right)+\mathrm{U}(\mathrm{x}, \mathrm{y}, \mathrm{z})=\text { constant }=\mathrm{E}\right.
$$

So we finally get,

$$
\frac{1}{\mathrm{~T}}\left(\frac{\mathrm{i} \overline{\mathrm{d}}(\mathrm{T})}{\mathrm{dt}}\right)=\mathrm{E}
$$

as the time value dependent equation and

$$
-\frac{\overline{\mathrm{h}}^{2}}{2 \mathrm{~m}}\left(\frac{1}{\mathrm{XYZ}}\left(\frac{\partial^{2}(\mathrm{XYZ})}{\partial\left(\mathrm{x}^{2} \mathrm{y}^{2} \mathrm{z}^{2}\right)}\right)+\mathrm{U}(\mathrm{x}, \mathrm{y}, \mathrm{z})=\mathrm{E}\right.
$$

as the time dependent equation.

Both equations 9 and 10 are solved using ordinary differential equations approaches. Applying separation of variables to Equation (9) and performing our integral calculus we get,

$$
\frac{1}{\mathrm{~T}}\left(\frac{\mathrm{i} \overline{\mathrm{h}} \mathrm{d}(\mathrm{T})}{\mathrm{dt}}\right)=\mathrm{E} \int \frac{1}{\mathrm{~T}} \mathrm{dT}=\int \frac{\mathrm{E}}{\mathrm{ih}} \mathrm{dt}
$$

Thus,

$$
\mathrm{T}=\mathrm{Be}^{\left(-\frac{\mathrm{i}}{\mathrm{h}}\right) \mathrm{Et}}
$$

This gives out time function which measures the time values of our stock assets from the time of release into the market up to any point of extinction. This is a very important corollary that should be taken with care as it describes the time dynamics associated with stock prices. The solution contains a complex variable $i$. The meaning and clarification of the solution in financial terms is shown on the plotted graph Figure 1. Notes on the interpretation should be well taken and considered. 


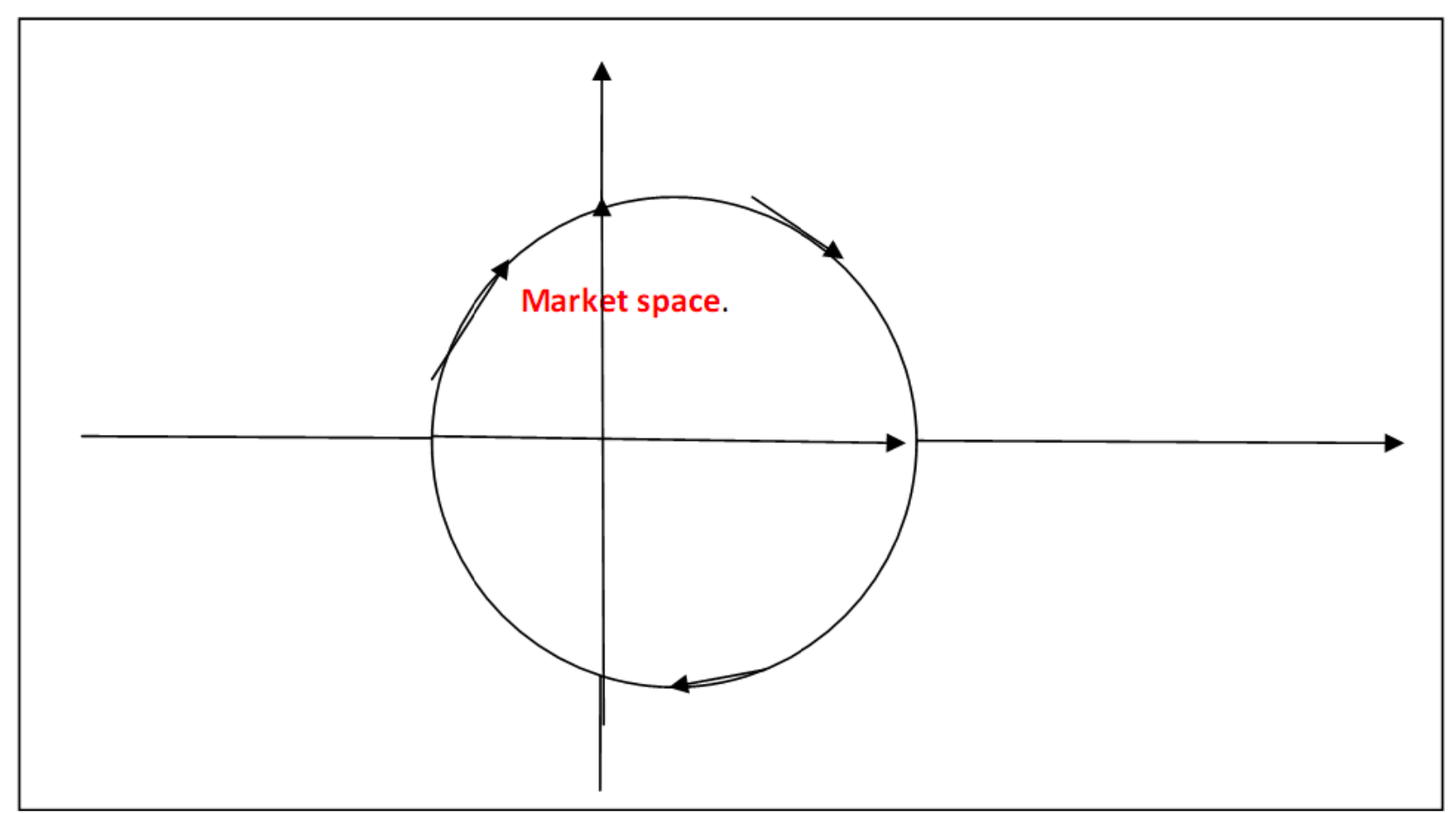

Figure 1. Time dynamics of stock prices. Source: Author's own compilations.

The figure above represents the time evolvements within the stock market. This means that stocks can evolve with time and again but within the market boundaries. The movement is controlled and restricted by market forces(both endogenous and exogenous).

The market space stock value function is obtained as,

$$
-\frac{\bar{h}^{2}}{2 m}\left(\frac{1}{X Y Z}\left(\frac{\partial^{2}(X Y Z)}{\partial\left(x^{2} y^{2} z^{2}\right)}\right)+U(x, y, z)=E(x, y, z)\right.
$$

The LHS is the Hamiltonian function. Thus, it should be well noted that, the SWE was built not only from Maxwell's equation but from the Hamiltonian as well. Thus we can safely write,

$$
H \overline{(X Y Z)}=E(X Y Z)
$$

This means that the Hamiltonian function is equal to energy. In financial terms, it means that the stock market price is equal to the market forces. This further implies that, it is the market forces which determine the stock prices. We shall further mathematically derive the solution:

Using (10) above, let $X Y Z$ and $x y z$ be equal to $P$ and $p$ respectively so that we get,

$$
-\frac{\bar{h}^{2}}{2 m}\left(\frac{1}{p}\right)\left(\frac{d^{2} P}{d p^{2}}\right)+U p=E p
$$

Equation (13) is a non-linear second order differential equation. We shall apply first make the equation linear and then solve the resultant using the auxiliary-equation formulations. So, the equation in linear form becomes,

$$
\left(\frac{d^{2} P}{d p^{2}}\right)-[U(p)+E(p)]\left(\frac{2 m p}{\bar{h}}\right)=0
$$

Thus, the auxiliary equation is obtained as: 


$$
v^{2}-\frac{2 m}{\bar{h}^{2}} p(U(p)+E(p))=0
$$

This gives,

$v^{2}-\frac{2 m}{\bar{h}^{2}}=0$ which is in quadratic form and the solutions obtained is given as,

$$
v= \pm \sqrt{\frac{2 m}{\bar{h}^{2}}}
$$

This gives,

$$
P=C_{1} e^{\sqrt{\frac{2 m}{\bar{h}^{2}}}}+C_{2} e^{-\sqrt{\frac{2 m}{\bar{h}^{2}}}}
$$

where $P=X Y Z$ (Stock market space value).

Solution (15) is our market space price function for our stock assets. $C_{1}$ and $C_{2}$ are arbitrary constants which can be obtained anytime given the initial market values (IMV). The solution tells us that, the market prices for stock assets are controlled and determined by the stock volume on trade and market money supply. Money supply is of importance as it determines the inflation factor which contributes much to the stock value. It should also be noted that the value of $v$ captures in the volatility factor given by $U$.

\section{Discussions}

This paper came through a phynance approach. We conjectured and proposed that stock price dynamics like waves, can be well modelled using Schrödinger's wave equation. We defined the SWE in financial terms and derived the time dependent Schrödinger Equation (TDSE) and time independent Schrödinger Equation (TISE). Further we procedurally derived the solutions for two forms. The two forms gave us the time function and market space value function for our stocks respectively. We found that, the Schrödinger Equation can better model stock price dynamics and give an estimation function for the stock prices. Given market values for factors such as stock volumes, money supply, initial stock prices, and market forces we can safely determine the market values of our stock assets and we can determine the time frequency, direction (clockwise/anticlockwise) and time intervals for stock dynamics within the market. The method of separation of variables and the auxiliary equation formulations are the basic methods used to derive our solutions. They proved to be competent. Therefore, phynance is an interesting cross breed that should be well considered. Physics models can well present the real life finance problems in a better and precise way. As such we highly recommend the adoption and application of such models.

\section{References}

Creedy, J., \& Martin, V. L. (Eds., 1994). Chaos and non-linear models in economics-theory and applications. Brookfield, Vermont: Edward Elgar Publ. Co.

Caginal, G., \& Desantis, M. (2009). Nonlinearity in the dynamics of financial markets. Nonlinear Analysis: Real World Applications, 12(2011), 1140-1151.

Christianto, V. (2014). A review of Schrödinger equation \& classical wave equation. Prespacetime Journal Published by QuantumDream, Inc. ISSN: 2153-8301

David, D. S. Y., \& Fatigun, A. T. (n.d.) The Schrodinger model and its applications. Available at https://www.academia.edu/2445611/The_Schrodinger_Model_and_its_Applications 
Erwin Schrödinger (1925) in Dronamraju, K. (1995). Erwin Schrödinger and the origins of molecular biology.

Faisal. (2016). Retrieved from https://www.quora.com/What-does-each-variable-mean-in-the-Schr\%C3\%B6dinger-equation

Hamilton, W, A. (1834). On a general method in dynamics. In Philosophical Transactions of the Royal Society Part II (pp. 247-330).

Heij, C., Schumacher, H., Hanzon, B., \& Praagman, K. (Eds., 1997). System dynamics in economic and financial models. Chichester, England: John Wiley \& Sons, Ltd.

Kunsch, P., Theys, M., Chevalier, A., \& Iacopetta, J. (2013). A system dynamics model of stock price movements. In Decision Making: Recent Developments and Worldwide Applications (pp.157-171).

Maxwell, J. C. (1892). A treatiseon electricity and magnetism. Oxford: Clarendon Press.

Ventana Systems, Inc. (1997). VENSIM 3.0 software, Harvard, MA. 